\title{
Natural scene categorization with minimal attention: Evidence from negative priming
}

\author{
SaChio OtsuKa AND Jun KawaguChi \\ Nagoya University, Nagoya, Japan
}

\begin{abstract}
Research has demonstrated that objects in natural scenes are categorized without the deployment of attention. However, in these types of studies, participants were required to directly respond to peripherally presented scenes, which might lead some participants to move their attention. If this is the case, the above conclusion concerning natural scenes may not be valid. We investigated this issue by using a negative priming (NP) paradigm in which participants did not directly respond to peripheral stimuli. Our results showed NP effect from ignored stimuli in natural scene categorization, but neither in letter discrimination (Experiment 1) nor in line-drawing categorization (Experiment 2). In addition, NP effects were observed even when probe stimuli were words (Experiments 3A and 3B). These findings suggest that people can categorize objects in natural scenes with minimal attention, that this process is specific to natural scenes, and that it is based on the semantic information of the images.
\end{abstract}

At times, individuals process simple geometrical figures, such as letters and lines, without focused attention, and the most popular explanation of such visual search is the feature integration theory (see, e.g., Treisman \& Gelade, 1980; Wolfe, Cave, \& Franzel, 1989). In this model, stimulus features such as lines, colors, and orientation are believed to be processed early, automatically, and in parallel. In this sense, all of the elemental bits of information in the display are present in the early stages of information processing. Feature information must then be recombined in order to recognize the objects in the display, and this latter process is assumed to require attention. In fact, many studies have demonstrated that discrimination tasks require attentional resources by using a dual-task paradigm in which interference is measured from concurrently performed attentionally demanding tasks (Braun, 1993, 1994; Braun \& Julesz, 1998; Braun \& Sagi, 1990; Lee, Koch, \& Braun, 1999; Sperling \& Dosher, 1986; Sperling $\&$ Melchner, 1978).

According to the feature integration theory, high-level visual processing, such as the categorization of complex stimuli in natural scenes, should require more attentional resources for processing. Recently, however, some researchers have reported that natural scenes could be processed rapidly in the visual system. Thorpe, Fize, and Marlot (1996) asked participants to detect animals in more than 4,000 natural images. They used a go/no-go categorization task in which participants were required to release the button if they saw an animal (go trials), or to keep their finger on the button otherwise (no-go trials). They measured accuracy, reaction time (RT), and event-related potentials (ERPs). The average proportion of correct responses was very high (94\%), and median RTs were very short (445 msec). Moreover, the ERP data showed a significant difference in amplitude approximately $150 \mathrm{msec}$ after the images were presented when animals were or were not present in natural images. These results indicated that individuals can rapidly categorize objects in natural images. Subsequent studies have reported similar results (BaconMacé, Macé, Fabre-Thorpe, \& Thorpe, 2005; Fize, FabreThorpe, Richard, Doyon, \& Thorpe, 2005; VanRullen \& Koch, 2003; VanRullen \& Thorpe, 2001a, 2001b).

If objects in natural scenes are rapidly categorized in the visual system, one needs to determine whether such categorization occurs with or without attention. The pioneer study exploring this issue was performed by $\mathrm{Li}$, VanRullen, Koch, and Perona (2002). Their experimental paradigm included three different conditions: a central condition, a peripheral condition, and a dual-task condition. In the central task condition, five randomly rotated letters were presented at the center of display, and participants were asked to determine whether these stimuli were the same (see Braun, 1994). The peripheral condition involved three different tasks: letter discrimination, colorpattern discrimination, and natural scene categorization. In the letter discrimination task, the stimulus was a randomly rotated letter "T" or "L," and the letter "L" was designated as target. Participants were required to detect the presence of the target letter. In the color-pattern discrimination task, the stimulus was a vertically bisected

S. Otsuka, s050307d@mbox.nagoya-u.ac.jp 
disk with a red half and a green half. Participants were asked to detect the presence of the target disk in which the red half was on the right side of the disk. In the natural scene categorization task, the stimulus was a complex natural image that included a target object that was either an animal or a vehicle. Participants were asked to detect and categorize the target object.

In the dual-task condition, participants were told to focus their attention on the central letters and respond to a peripheral stimulus as fast as possible before responding to the central letters. In Li et al. (2002), a training session consisted of about 12,000 trials of all different tasks and took more than $10 \mathrm{~h}$ for each participant.

The results of Li et al. (2002) showed that the performance on the natural scene categorization task was similar both in the single-task condition and in the dual-task condition, and participants could categorize animals or vehicles equally well. In contrast, performance was at chance level on the letter discrimination and color-pattern discrimination tasks in the dual-task condition. Li et al. concluded that the categorization of natural scenes requires very little attention, and higher level representations can be accessed in the near absence of attention, in contrast to letters and color patterns.

However, an alternative explanation of their data is that because their participants were required to respond directly to peripheral images, they may have shifted attention to them efficiently. It is critical that Li et al. (2002) did not clearly explain why they were certain that their participants did not pay attention to the peripheral stimuli, despite directly responding to the peripheral stimuli. Although they claimed that it was not possible for the participants to shift attention, we think that this direct response to the peripheral stimuli still leaves the possibility that participants attended to them. Moreover, because of the massive training, their participants might merely have learned to shift attention between the central letters and peripheral stimuli more rapidly and efficiently in comparison with the participants who received less training. In other words, it is possible that their conclusion that rapid natural scene categorization occurs in the near absence of attention is made on the basis of the increased efficiency of their participants' response strategies.

If this is the case, participants who paid attention to peripheral images would be expected to perform categorization just as well during the dual-task condition as they did during the single-task condition. The participants of Li et al. (2002) were likely experts in shifting attention given the massive training, which could account for the high proportion of "attended" trials. If this is true, then $\mathrm{Li}$ et al.'s conclusion that the categorization of target objects in natural scenes is accomplished in the near absence of attention has not been demonstrated by their experimental procedure. To adequately address the issue of the attentional demands of categorization in natural scenes, we need to employ an experimental design in which participants are not given massive training and are not required to respond directly to peripheral stimuli. ${ }^{1}$ The present set of experiments examines whether target objects in natural scenes are categorized without the deployment of atten- tion using a negative priming (NP) paradigm (cf. Lavie \& Fox, 2000). ${ }^{2}$ We employed two attention-load conditions (low and high) for prime, and participants carried out the same response across the two conditions, in which the amount of attentional resources allocated to prime differed. Participants performed a letter discrimination task and a natural scene categorization task in Experiment 1 in order to examine the advantage of natural scenes over letters. In Experiment 2, we adopted a line-drawing categorization task and compared participants' performances on object categorization in natural scenes versus mere object categorization to examine whether categorization with minimal attentional resources was specific to natural scenes. In Experiments 3A and 3B, we used word stimuli in the probe display to test whether object categorization in natural scenes with minimal attention was based on the semantic information of natural images.

\section{EXPERIMENT 1}

In Experiment 1, we investigated whether or not object categorization in natural scenes and letter discrimination are performed with minimal attentional resources. In our study, we employed an NP paradigm (Lavie \& Fox, 2000) in which participants did not need to respond to peripheral stimuli themselves. We manipulated the attentional load (low and high) at the center of the display, following the methodology of Lavie and Fox. Lavie and Fox found that

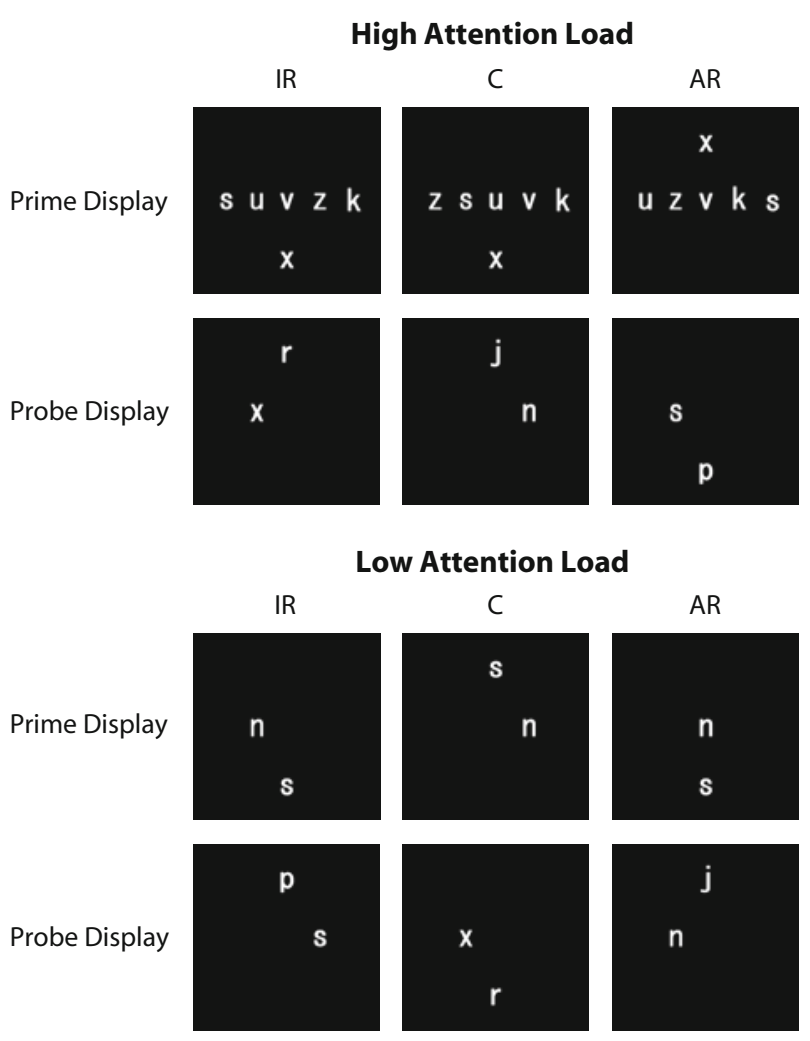

Figure 1. Sample displays from the high- and low-attentionload conditions in the letter discrimination task. IR, ignored repetition; $C$, control; AR, attended repetition. 
NP was influenced by attentional resources allocated to the prime display, and that it was eliminated when the set size for the visual search was larger than about four items, because the larger set size exhausted resources for the searched center stimuli. Thus, we utilized set sizes of one and five items for the low- and high-attention-load conditions, respectively. This procedure allowed us to determine whether the peripheral stimulus, which participants minimally attend to, is processed. Moreover, VanRullen, Reddy, and Koch (2004) proposed that objects in natural scenes do not pop out in a visual search task, whereas they could be discriminated from distractors in the near absence of attention with a dual-task paradigm. Thus, in this study, we would be able to expect the influence of attention load with natural scenes and to determine whether object categorization in natural scenes is or is not performed with minimal attention.

If natural scenes are categorized with minimal attention, then the NP effect should be observed in the natural scene categorization task, irrespective of the attention-load condition. If the natural scene categorization in the near absence of attention reported by $\mathrm{Li}$ et al. (2002) is based on their participants' more efficient response strategies given their massive training, we should not observe an NP effect in the high-attention-load condition, although it should be present in the low-attention-load condition. We should not observe NP in the letter-discrimination task under the high-attention-load condition if more resources are needed to process letters.

\section{Method}

Participants. Twelve graduate and undergraduate students of Nagoya University ( 7 men and 5 women, age range 20-32 years) participated in this experiment. All were right-handed and reported normal or corrected-to-normal visual acuity and normal color vision. They all volunteered for participation in this study and were naive as to the purpose of this study.

Apparatus. This experiment was controlled by an AV tachistoscope (IWATSU ISEC, IS-7301). Visual stimuli were presented on a color monitor (Mitsubishi, RDF221H). The refresh rate of the monitor was $50 \mathrm{~Hz}$. Image resolution was $1,024 \times 1,024$ pixels. Participants were seated in a dimly lit room at $80 \mathrm{~cm}$ from the computer screen. Participants' responses were measured using the response unit of the AV tachistoscope (IS-7211).

Letter stimuli. Figure 1 shows examples of the prime and probe displays in the high- and low-attention-load conditions for the letter discrimination task. Each trial consisted of a prime display and a probe display. The magnitude of the priming effect from the irrelevant prime distractor was assessed on the subsequent probe display. Letter stimuli were presented in white on a black background. We utilized set sizes of one or five stimuli for the low- and high-attentionload conditions, respectively, because Lavie and Fox (2000) found that NP was eliminated for a set size larger than four.

The target letters were $n, s$, or $x$, and the nontargets of searched letters in the high-attention-load condition were $k, u, v$, and $z$. All the letters were written in lowercase in the Gothic font. Each letter subtended a visual angle of about $1.4^{\circ}$ vertically and about $1.4^{\circ}$ horizontally. In the low-attention-load condition, a target letter and a distractor letter appeared, and none of the searched letters was presented in the prime display. A target letter was presented at one of five equally probable positions. Distractor letters presented in the prime display changed with the three priming conditions. In the high-attention-load condition, a target letter was accompanied by four nontarget letters that had no association with any response on
Animal
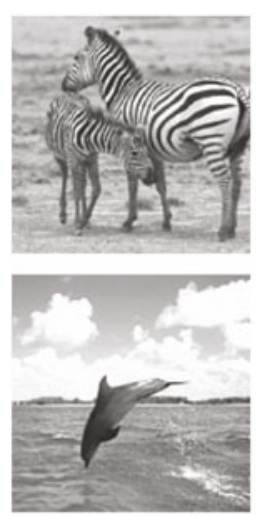

Nontargets
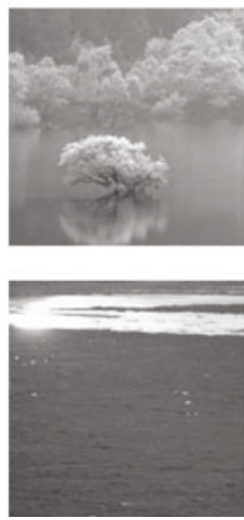

Vehicle
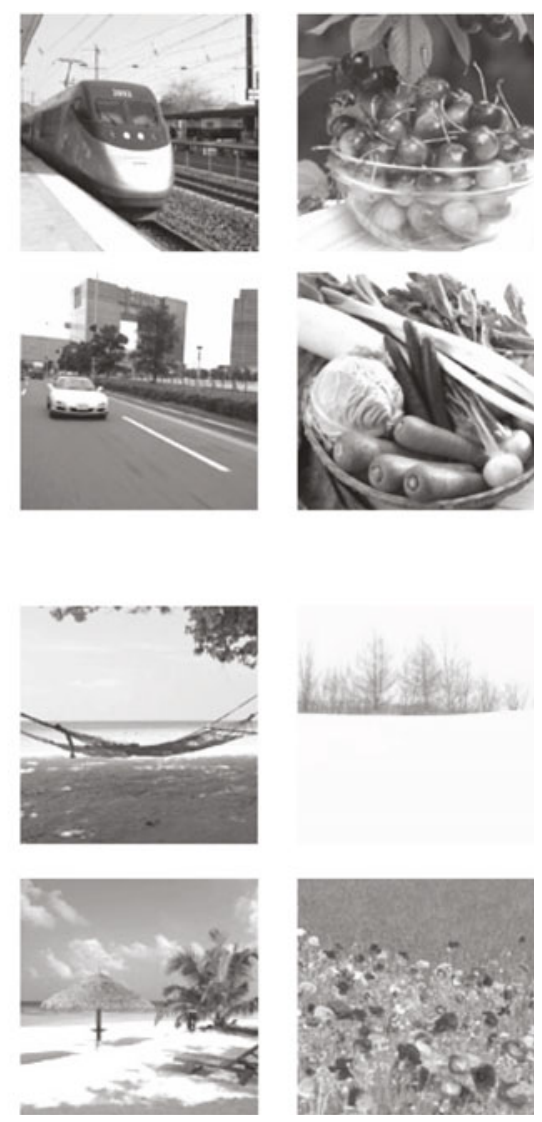

Figure 2. Examples of target pictures (animal, vehicle, and food) and nontarget pictures used in the natural scene categorization task.

the prime display. On the prime display, all the distractors were incompatible with the response required for the current target, and all appeared randomly and equally either above or below the center of the screen. The eccentricity between each searched stimulus was on average $3.2^{\circ}$, so that the eccentricity between the letter presented at the center and at either end of the letters was about $6.4^{\circ}$ and the eccentricity between both ends of the letters was about $12.8^{\circ}$. Also, the eccentricity between the ignored distractor letter presented above or below the center of the screen and the central letter was about $4.3^{\circ}$, so that the eccentricity between the ignored distractor letter and either of the next letters to the central letter was about $5.2^{\circ}$ and the eccentricity between the ignored distractor letter and either end of the letters was about $7.7^{\circ}$.

In both the low-attention-load condition and the high-attentionload condition, the target letter on the probe display appeared in one of two central locations on the immediate left or right of fixation, with one distractor $(j, p$, or $r)$ that had no associated response in this experiment. The distractors were never presented in the same position across the prime and probe displays. Also, all the letters were equiprobably presented multiple times across the trials.

There were three priming conditions. In the attended repetition (AR) condition, the probe display contained a target that was identical to the target in the prime display. The distractor letters always differed between prime and probe displays (e.g., a target $s$ with a distractor $x$, followed by a target $s$ with a distractor $p$ ). In the control (C) condition, the probe display contained target and distractor letters that were different from those in the prime display (e.g., a target $s$ with a distractor $x$, followed by a target $n$ with a distractor $j$ ). In the 

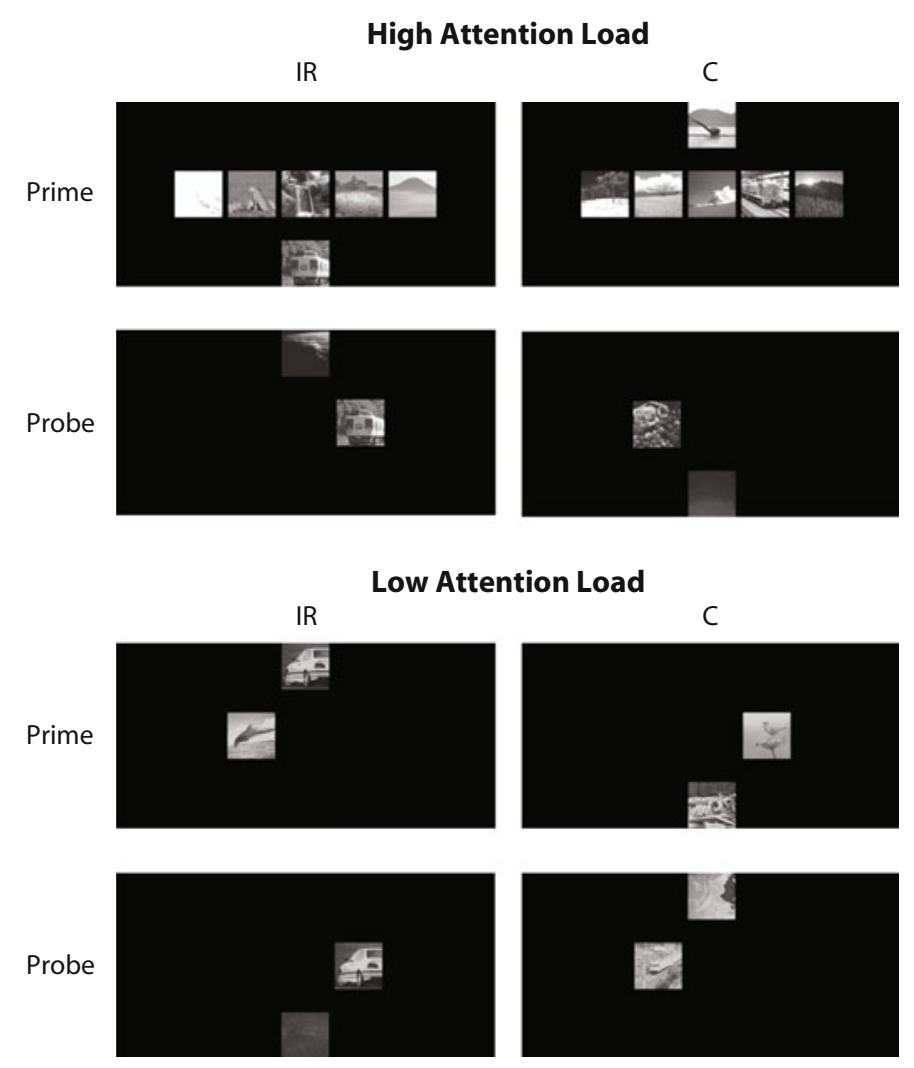

Figure 3. Sample displays from the high- and low-attention-load conditions in the natural scene categorization task. IR, ignored repetition; $C$, control. Sample displays in the AR condition were omitted.

ignored repetition (IR) condition, a target on the probe display had the same identity as the distractor in the previous prime display (e.g., a target $s$ with a distractor $x$, followed by a target $x$ with a distractor $r$ ). NP was measured by subtracting the observed data in the IR condition from the corresponding data in the $\mathrm{C}$ condition.

In line with the procedure of Lavie and Fox (2000), we included the AR condition because the previous studies of NP have found that NP is generally larger when a proportion of trials included repetition of the target item across the prime and probe displays (Kane, May, Hasher, Rahhal, \& Stoltzfus, 1997; Lavie \& Fox, 2000). However, the data of IR and AR were analyzed separately because the AR condition was not critical to this experiment (see Lavie \& Fox, 2000).

Natural scene stimuli. Examples of target and nontarget stimuli used in the natural scene categorization task are presented in Figure 2. A total of 2,304 color images were selected from commercially available CD-ROMs of photographs. The images were chosen to be as varied as possible. The categories of target objects were animals (336 images), vehicles (336 images), and food (336 images). Target images contained at least one target object. The animal category images included fish, mammals, birds, insects, reptiles, and so on. The vehicle category images contained electric trains, airplanes, ships, cars, and so on. The food category images included fruits, grains, vegetables, and so on. Searched nontargets in the high-attentionload condition were natural landscapes and city scenes that did not contain any object belonging to the three target categories (864 images). Distractor images on the probe display contained space, planets, and starlit skies (432 images) that were not associated with any response. Participants had no knowledge about the size, position, or number of targets in a single image, and the natural images were never presented more than once except for the critical stimulus in the IR condition and the target in the AR condition within a certain trial. Each image subtended a visual angle of about $2.9^{\circ}$ vertically and about $2.9^{\circ}$ horizontally.

In the natural scene categorization task, these pictures were arranged similarly to the stimulus arrangement of the letter discrimination task (see Figure 3). We presented five searched images and one distractor image on the prime display in the high-attention-load condition. The five searched images always included one target image that belonged to the categories of animal, vehicle, or food, and four nontarget images that were natural landscapes and city scenes. In the low-attention-load condition, the display included one target image and one distractor image, and none of the searched images was presented in the prime display. In the AR condition, the probe display contained a target image that was identical to the target image in the prime display. The distractor images always differed between the prime and probe displays (e.g., a target food image with a distractor vehicle image, followed by a target food image with a distractor space image). In the $\mathrm{C}$ condition, the probe display contained a target and distractor images that were different from those in the prime display (e.g., a target vehicle image with a distractor animal image, followed by a target food image with a distractor starlit skies image). In the IR condition, a target in the probe display had the same identity as the distractor in the previous prime display (e.g., a target animal image with a distractor vehicle image, followed by a target vehicle image whose identity was the same as that of the distractor in the prime display, presented with a distractor planet image). We created six lists of natural images to facilitate counterbalancing across participants (e.g., some images that were presented for one participant in the IR and high-attention-load condition were presented for another participant in the $\mathrm{C}$ and low-attention-load condition). In addition, we selected 576 color images for the practice trials $(84$ images for animal category, 84 images for vehicle category, 84 images for food category, 
216 images for searched nontargets, and 108 images for distractors on the probe display) that were not presented in the experimental trials.

Design. The manipulated variables were target task (letter discrimination and natural scene categorization), attention load (low and high), and relationship between the prime and probe displays (IR, C, and AR). All variables were within-subjects factors.

Procedure. The experiment was divided into 16 blocks for each target task, with each block comprising 27 trials. The relationship between the prime and probe displays was randomly presented within blocks ( $\mathrm{IR}=9$ trials, $\mathrm{C}=9$ trials, and $\mathrm{AR}=9$ trials). Within each block, the three target stimuli were equally presented for each task. Each participant received 432 trials for each task, for a total of 864 trials. Participants performed each task on different days. The order of attention load and target task was counterbalanced across participants. Participants were required to respond to the target stimulus by pressing a key with their dominant hand (e.g., in the letter discrimination task, participants indicated the target letter $n$ with the index finger, the target letter $s$ with the middle finger, and the target letter $x$ with the ring finger, and in the natural scene categorization task, participants indicated the target image animal with the index finger, the target image vehicle with the middle finger, and the target image food with the ring finger). The arrangement of the keys was counterbalanced across participants.

Following the instructions for this experiment, participants performed 108 practice trials for each task that were divided into four blocks consisting of two high- and two low-attention-load conditions. The low- and high-attention-load conditions were presented in separate blocks. Half the participants received the high-attention-load blocks first, followed by the low-attention-load blocks for each task. The remaining participants received the blocks in the reverse order.

Figure 4 shows the trial schedule of the experiment. A fixation cross was presented at the center of the computer screen for $1,000 \mathrm{msec}$. This was immediately replaced by the prime display, which appeared for $240 \mathrm{msec}$. Participants used the button box keys of the tachistoscope to make their responses. They were required to respond to a target letter (in the letter discrimination) or a target object in an image (in the natural scene categorization) by pressing the keys as indicated above. Following the response to the prime display, a blank screen was presented for $300 \mathrm{msec}$, then the probe display was presented for $240 \mathrm{msec}$. The task for the probe display was the same as that for the prime display. Participants used the same response-key mapping both for the prime display and for the probe display. Following their response to the probe display, there was an intertrial interval of $1,000 \mathrm{msec}$ before the next trial began. In the letter discrimination task, participants were asked to discriminate a target letter within the searched letters of the prime and probe displays. In the natural scene categorization task, they were required to categorize a target object in a natural scene as either animal, vehicle, or food. Importantly, participants were instructed to ignore the stimulus presented randomly above or below the center of the screen (i.e., the distractor)

\section{Results and Discussion}

The mean RTs and error rates as a function of the experimental conditions are presented in Table 1 for the prime display and Table 2 for the probe display. Trials

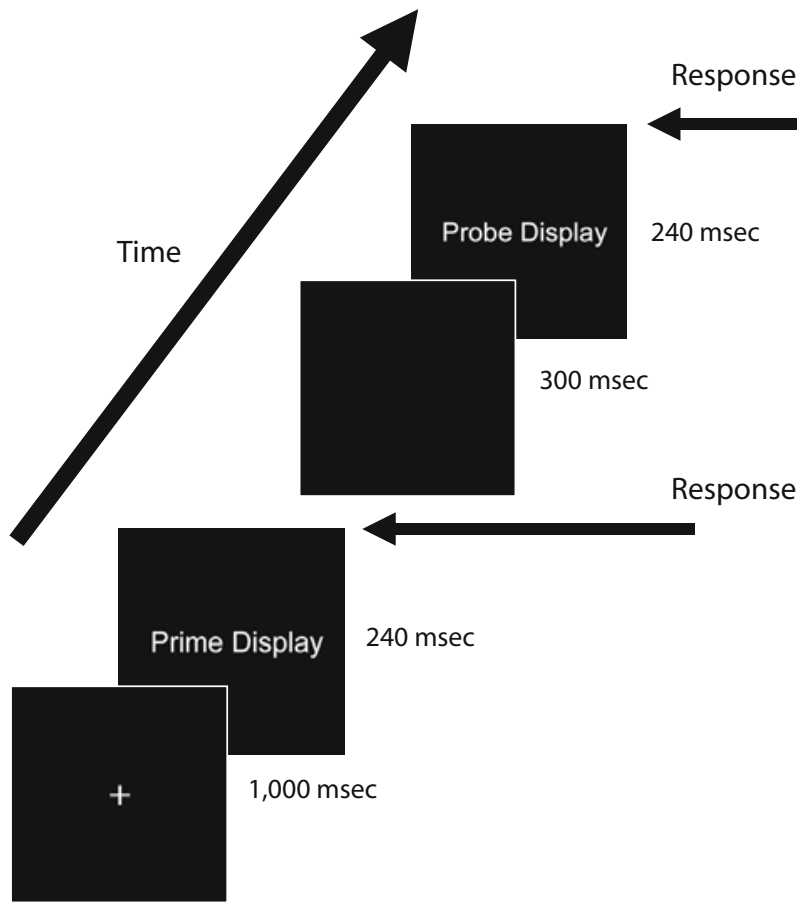

Figure 4. Trial schedule in Experiment 1. The time schedule of trials was identical both in the letter discrimination task and in the natural scene categorization task.

with errors and trials preceded by an error on the prime display were excluded from the probe RT analysis, as were responses that deviated $2 S D$ s from an individual participant's means. We separately analyzed the data of the letter discrimination and natural scene categorization.

Letter discrimination. First, we analyzed the data of the prime display. A one-way within-subjects ANOVA was performed on the mean correct RTs and on the mean error rates, with the factor being attention load (low vs. high). The RT analysis showed a significant main effect of attention load $\left[F(1,11)=110.28, M S_{\mathrm{e}}=3,230.85, p<.01\right]$. The error rate analysis also revealed a significant main effect of attention load $\left[F(1,11)=12.59, M S_{\mathrm{e}}=21.72, p<\right.$ $.01]$. These results show that attention load was effectively manipulated in the target processing of the prime display, with poor performance under the high-attention-load condition, as expected.

Next, we analyzed the data of the probe display. A twoway (attention load: low vs. high; and the critical relationship between the prime and probe displays: IR vs. C)

Table 1

Mean Reaction Times (RTs, in Milliseconds), Standard Errors, and Percentages of Error (PEs) in the Prime Display As a Function of Each Experimental Condition in Experiment 1

\begin{tabular}{|c|c|c|c|c|c|c|c|c|}
\hline \multirow[b]{3}{*}{ Type of Target Task } & \multicolumn{8}{|c|}{ Attention Load } \\
\hline & \multicolumn{4}{|c|}{ Low } & \multicolumn{4}{|c|}{ High } \\
\hline & RT & $S E$ & $\mathrm{PE}$ & $S E$ & RT & $S E$ & $\mathrm{PE}$ & $S E$ \\
\hline Letter discrimination & 519 & 12 & 4.1 & 1.0 & 763 & 20 & 10.8 & 2.0 \\
\hline Natural scene categorization & 659 & 17 & 8.1 & 2.1 & 841 & 18 & 26.4 & 2.8 \\
\hline
\end{tabular}


Table 2

Mean Reaction Times (RTs, in Milliseconds), Standard Errors, and Percentages of Error (PEs) in the Probe Display As a Function of Each Experimental Condition in Experiment 1

\begin{tabular}{|c|c|c|c|c|c|c|c|c|c|c|c|c|c|c|c|c|}
\hline \multirow[b]{3}{*}{ Condition } & \multicolumn{8}{|c|}{ Letter Discrimination } & \multicolumn{8}{|c|}{ Natural Scene Categorization } \\
\hline & \multicolumn{4}{|c|}{ Low } & \multicolumn{4}{|c|}{ High } & \multicolumn{4}{|c|}{ Low } & \multicolumn{4}{|c|}{ High } \\
\hline & RT & $S E$ & $\mathrm{PE}$ & $S E$ & RT & $S E$ & $\mathrm{PE}$ & $\overline{S E}$ & RT & $S E$ & $\mathrm{PE}$ & $\overline{S E}$ & RT & $S E$ & $\mathrm{PE}$ & $S E$ \\
\hline IR & 493 & 15 & 5.3 & 1.4 & 487 & 16 & 3.0 & 0.6 & 643 & 15 & 7.5 & 1.7 & 640 & 14 & 5.3 & 1.5 \\
\hline $\mathrm{C}$ & 473 & 14 & 5.0 & 1.1 & 488 & 14 & 2.7 & 0.8 & 606 & 15 & 6.0 & 1.3 & 613 & 18 & 6.0 & 0.9 \\
\hline $\mathrm{AR}$ & 425 & 14 & 2.9 & 0.8 & 446 & 13 & 0.3 & 0.2 & 455 & 15 & 0.4 & 0.3 & 503 & 17 & 1.9 & 0.9 \\
\hline $\mathrm{C}-\mathrm{IR}$ & $-20^{* *}$ & & -0.3 & & 1 & & -0.3 & & $-37^{* *}$ & & -1.5 & & $-27^{* *}$ & & 0.7 & \\
\hline
\end{tabular}

within-subjects ANOVA on the mean correct RTs indicated that both the effect of the relationship and the interaction between attention load and the relationship were significant $\left[F(1,11)=10.75, M S_{\mathrm{e}}=99.89, p<.01\right.$, and $F(1,11)=18.46, M S_{\mathrm{e}}=76.34, p<.01$, respectively $]$. The effect of attention load was not significant $(F<1)$. An examination of the simple main effects revealed that the effect of relationship was significant only in the lowattention-load condition $\left[F(1,11)=32.59, M S_{\mathrm{e}}=75.83\right.$, $p<.01]$. Thus, we observed a -20 -msec NP effect. A similar ANOVA on the mean error rates did not reveal any significant results. As such, on the basis of the RT data, our results are consistent with the findings of Lavie and Fox (2000). They argued that the presence of the significant NP in the low-attention-load condition was due to the participants' abilities to allocate their resources not only to the centrally presented letter, but also to the peripherally presented letter. Meanwhile, in the high-attention-load condition, participants could not allocate their resources to the peripheral letter because they exhausted most of their resources by processing the centrally presented letters. This exhaustion of resources would similarly account for our results.

Natural scene categorization. As with the letter discrimination task, we first analyzed the data of the prime display. A one-way within-subjects ANOVA was performed on the mean correct RTs and on the mean error rates with the attention load (low vs. high) factor. The RT data revealed a significant main effect of attention load $\left[F(1,11)=24.60, M S_{\mathrm{e}}=8,022.78, p<.01\right]$. The error data also showed a robust effect of attention load $\left[F(1,11)=173.22, M S_{\mathrm{e}}=11.58, p<.01\right]$. As with the letter discrimination task, these results demonstrate that attention load was effectively manipulated as we expected.

Next, we analyzed the data of the probe display. A twoway within-subjects ANOVA on the mean correct RTs with two factors being attention load (low vs. high) and the critical relationship between the prime and probe displays (IR vs. C) indicated that the effect of the relationship was significant $\left[F(1,11)=13.27, M S_{\mathrm{e}}=933.60, p<\right.$ $.01]$. However, neither the effect of attention load nor the interaction between attention load and relationship were significant $\left(F_{\mathrm{S}}<1\right)$, thus showing significant NP effects both in the low-attention-load condition and in the highattention-load condition ( $-37 \mathrm{msec}$ and $-27 \mathrm{msec}$, respectively). This result is consistent with our hypothesis that natural scene categorization is accomplished with minimal attentional resources. However, a similar ANOVA on the error rates did not show any significant effect.

Thus, our findings suggest that people can categorize target objects in natural scenes with minimal attention. On the other hand, people need attentional resources to discriminate letters, despite the belief that letters are simpler stimuli than natural scenes. Our results are consistent with the finding of the previous study documenting the advantage of natural scene categorization (Li et al., 2002), and, importantly, this advantage was confirmed using an experimental paradigm that did not have the confounds of prior study (i.e., the possibility of attending to peripheral stimuli, and the possibility of increased attentional efficiency because of massive training).

We also performed analyses on the AR condition, running a two-way within-subjects ANOVA with attention load (low vs. high) and relationship (AR vs. C) as the two factors. The RT data showed significant effects of relationship both in the letter discrimination task and in the natural scene categorization task $[F(1,11)=83.24$, $M S_{\mathrm{e}}=293.70, p<.01$, and $F(1,11)=98.73, M S_{\mathrm{e}}=$ $2,065.57, p<.01$, respectively]. These effects were also replicated with the error rate data $[F(1,11)=11.01$, $M S_{\mathrm{e}}=5.37, p<.01$, and $F(1,11)=30.51, M S_{\mathrm{e}}=9.09$, $p<.01$, respectively]. These results could be interpreted as being typical positive repetition priming effects, but are inconsistent with the previous findings (Johnston \& Dark, 1986; Lavie, 1997; Lavie \& Fox, 2000), since they show greater positive priming from AR targets with higher load. Although we could not explain the incompatibility between our findings and the previous results, the results of these analyses are not critical for the interpretation of the NP effects observed and will not be discussed in the following experiments.

\section{EXPERIMENT 2}

We found that target objects in natural scenes could be categorized with minimal attention in Experiment 1. However, some psychologists wonder whether object categorization with minimal attention is specific to natural scenes. It may be possible that people can also categorize target objects of simpler stimuli (such as line drawings) and not just stimuli in natural scenes, irrespective of attention load. In Experiment 2, we therefore examined this possibility using a line-drawing categorization task with 
Animal
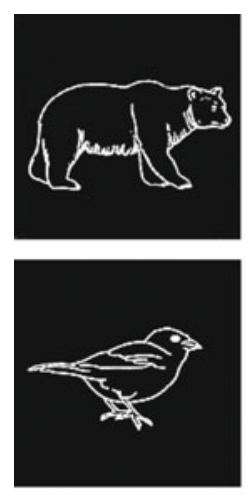

Nontargets
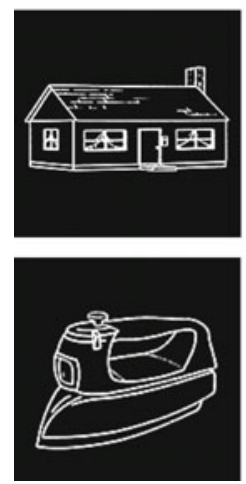

Figure 5. Examples of target stimuli (animal, vehicle, and food) and nontarget stimuli used in the line-drawing categorization task.

the same manipulations and procedures as those used in Experiment 1. If object categorization with minimal attention is specific to natural scenes, then NP should not be observed with line drawings in the situation in which participants cannot use their attentional resources. If people can simply categorize target objects belonging to certain categories with minimal attentional resources, regardless of whether they are natural scenes or line drawings, we will observe NP with line drawings as well as natural scenes, regardless of attention load.

Also, in Experiment 1, half of the participants performed the natural scene categorization following letter discrimination, so their performances of natural scene categorization might be contaminated by doing the letterdiscrimination task first. Thus, to examine the exact ad- vantage of natural scene, in Experiment 2, target task (line-drawing categorization and natural scene categorization) was set as a between-subjects factor.

\section{Method}

Participants. Twenty-four graduate and undergraduate students of Nagoya University ( 8 men and 16 women, age range $18-27$ years) participated in Experiment 2. All were right-handed and reported normal or corrected-to-normal visual acuity and normal color vision. None had participated in Experiment 1. They were divided into two groups for each task.

Apparatus and Stimuli. The apparatus was identical to that of Experiment 1.

The line-drawing stimuli were selected from the stimuli used by Snodgrass and Vanderwart (1980); examples are presented in Figure 5. Line drawings were printed in white on a black background. Each stimulus subtended a visual angle of about $2.6^{\circ}$ vertically and about $2.1^{\circ}$ horizontally. We selected nine stimuli for each target category. As in Experiment 1, the target categories were animals, vehicles, and food. The nontargets that had to be searched on the prime display were 108 objects of literary tools, furniture, and so on, and never belonged to the target categories. The distractors presented on the probe display that were irrelevant to the task were 9 objects belonging to the category of musical instruments (e.g., a piano, a trumpet, and a classic guitar) both in the low-attention-load condition and in the high-attention-load condition. These line drawings were equiprobably presented multiple times across the trials. All of the target stimuli were equiprobably presented in all the attention load conditions and priming conditions for each participant. The arrangement of the stimuli was the same as that of Experiment 1 .

In the natural scene categorization task, the natural images and the arrangement of the images were identical to those of Experiment 1.

Design. The variables that were manipulated were target task (line-drawing categorization and natural scene categorization), attention load (low and high), and the relationship between prime display and probe display (IR, C, and AR). Only the target task was a between-subjects factor.

Procedure. Each target task comprised 16 blocks of 27 trials each, so that each participant completed 432 trials. The attentionload conditions were presented in separate blocks. The order of the attention-load levels was counterbalanced across participants. Following the task introduction, participants performed 108 practice trials that were divided into four blocks that consisted of two highand two low-attention-load conditions. Participants were asked to categorize the target objects by pressing the keys that were assigned to each target category type (animal, vehicle, and food). The trial schedule was the same as that of Experiment 1.

\section{Results and Discussion}

The mean RTs and error rates as a function of the experimental conditions are presented in Table 3 for the prime display and Table 4 for the probe display. Trials with errors and trials preceded by an error on the prime display were excluded from the probe RT analysis, as were responses that deviated $2 S D$ s from an individual partici-

Table 3

Mean Reaction Times (RTs, in Milliseconds), Standard Errors, and Percentages of Error (PEs) in the Prime Display As a Function of Each Experimental Condition in Experiment 2

\begin{tabular}{|c|c|c|c|c|c|c|c|c|}
\hline \multirow[b]{3}{*}{ Type of Target Task } & \multicolumn{8}{|c|}{ Attention Load } \\
\hline & \multicolumn{4}{|c|}{ Low } & \multicolumn{4}{|c|}{ High } \\
\hline & RT & $S E$ & $\mathrm{PE}$ & $S E$ & RT & $S E$ & $\mathrm{PE}$ & $S E$ \\
\hline Line-drawing categorization & 582 & 13 & 4.5 & 0.9 & 805 & 19 & 14.8 & 2.2 \\
\hline Natural scene categorization & 682 & 21 & 7.6 & 1.7 & 807 & 21 & 24.7 & 2.4 \\
\hline
\end{tabular}


pant's means. Data were analyzed separately for the linedrawing categorization and natural scene categorization.

Line-drawing categorization. For the prime data, a one-way (attention load: low vs. high) within-subjects ANOVA was conducted. The RTs and error rates analyses revealed significant main effects of attention load $\left[F(1,11)=186.94, M S_{\mathrm{e}}=1,594.34, p<.01\right.$, and $F(1,11)=$ $39.05, M S_{\mathrm{e}}=16.30, p<.01$, respectively]. These analyses show that attention load was effectively manipulated as expected.

For RTs of the probe display, a two-way (attention load: low vs. high; and the critical relationship between the prime and probe displays: IR vs. C) within-subjects ANOVA revealed that the main effect of relationship was significant $\left[F(1,11)=13.41, M S_{\mathrm{e}}=220.37, p<.01\right]$. In addition, the ANOVA of the RTs showed a significant interaction $\left[F(1,11)=13.04, M S_{\mathrm{e}}=258.67, p<.01\right]$. Examination of the simple main effects revealed that the NP effect was found only in the low-attention-load condition $(-32 \mathrm{msec})$ $\left[F(1,11)=16.64, M S_{\mathrm{e}}=379.74, p<.01\right]$. No significant effects emerged from a similar ANOVA on the error rates. As expected, on the basis of the results of RTs, NP was not observed in the high-attention-load condition as was found in the letter discrimination task. These results suggest that object categorization requires more than minimal attention when the stimuli are line drawings.

Two-way ANOVAs on RTs and error rates with the factors of attention load (low vs. high) and relationship (AR vs. C) showed significant effects of relationship $[F(1,11)=$ $47.11, M S_{\mathrm{e}}=3,473.38, p<.01$, and $F(1,11)=26.22$, $M S_{\mathrm{e}}=21.46, p<.01$, respectively]. These results indicate repetition priming effects.

Natural scene categorization. An ANOVA showed the same patterns as those found in Experiment 1 both for the prime display and for the probe display. Both RT and error rate data showed the significant effects of attention load in the prime display $\left[F(1,11)=38.60, M S_{\mathrm{e}}=\right.$ $2,430.82, p<.01$, and $F(1,11)=142.74, M S_{\mathrm{e}}=12.31$, $p<.01$, respectively]. Also, the RT data of the probe display indicated that the effect of relationship was significant $\left[F(1,11)=11.84, M S_{\mathrm{e}}=653.33, p<.01\right]$, but neither the effect of attention load nor the interaction between attention load and relationship were significant $\left(F_{\mathrm{S}}<1\right)$. No significant effects were found on a similar ANOVA that was performed on the error rates. As was the case for natural scene categorization in Experiment 1, the NP effects were replicated both in the low-attention-load con- dition and in the high-attention-load condition ( $-24 \mathrm{msec}$ and $-27 \mathrm{msec}$, respectively). These results suggest that the NP effects found in the natural scene categorization task in Experiment 1 are not due to the prior implementation of similar task (e.g., letter discrimination). More importantly, the results of Experiment 2 suggest that object categorization with minimal attention is specific to natural scenes.

Two-way ANOVAs on RTs and error rates with the variables of attention load (low vs. high) and relationship (AR vs. C) revealed significant repetition priming effects $\left[F(1,11)=95.79, M S_{\mathrm{e}}=2,284.87, p<.01\right.$, and $F(1,11)=29.52, M S_{\mathrm{e}}=11.51, p<.01$, respectively].

\section{EXPERIMENT 3A}

In Experiment 3A, we investigated whether object categorization in natural scenes with minimal attention was made on the basis of the low-level features of the stimuli or on semantic information about the natural images. Because target images in the probe display were identical to ignored images in the prime display, the NP effects observed in the natural scene categorization task could be accounted for by the extraction of low-level features without the extraction of semantic information. To address this issue, we replaced the natural images presented in the probe display with the written word that best described the target object. If semantic information of natural images that are presented peripherally in the prime display is processed irrespective of attention, NP would be expected to be observed with a word probe both in the low-attentionload condition and in the high-attention-load condition. If semantic information of natural images that are presented peripherally is not processed at all and NP effects observed in the natural scene categorization task in Experiments 1 and 2 are based only on the low-level features that do not contain semantic information, NP would not be expected to be observed with the word stimuli.

\section{Method}

Participants. Twelve native Japanese undergraduate students of Nagoya University ( 3 men and 9 women, age range $18-31$ years) participated in Experiment 3A. All were right handed and reported normal or corrected-to-normal visual acuity and normal color vision. None had participated in either Experiments 1 or 2.

Apparatus and Stimuli. The apparatus was identical to that of Experiment 1.

The natural images presented in the prime display were the same as those presented in Experiment 1.

Table 4

Mean Reaction Times (RTs, in Milliseconds), Standard Errors, and Percentages of Error (PEs) in the Probe Display As a Function of Each Experimental Condition in Experiment 2

\begin{tabular}{|c|c|c|c|c|c|c|c|c|c|c|c|c|c|c|c|c|}
\hline \multirow[b]{3}{*}{ Condition } & \multicolumn{8}{|c|}{ Line-Drawing Categorization } & \multicolumn{8}{|c|}{ Natural Scene Categorization } \\
\hline & \multicolumn{4}{|c|}{ Low } & \multicolumn{4}{|c|}{ High } & \multicolumn{4}{|c|}{ Low } & \multicolumn{4}{|c|}{ High } \\
\hline & RT & $S E$ & $\mathrm{PE}$ & $S E$ & $\mathrm{RT}$ & $S E$ & $\mathrm{PE}$ & $S E$ & RT & $S E$ & $\mathrm{PE}$ & $S E$ & RT & $S E$ & $\mathrm{PE}$ & $S E$ \\
\hline IR & 565 & 18 & 7.0 & 1.5 & 553 & 15 & 7.0 & 1.2 & 631 & 12 & 7.9 & 1.5 & 636 & 15 & 7.0 & 1.3 \\
\hline $\mathrm{C}$ & 533 & 16 & 7.6 & 1.7 & 554 & 14 & 7.8 & 1.7 & 607 & 14 & 7.3 & 1.6 & 609 & 21 & 6.8 & 0.8 \\
\hline AR & 415 & 14 & 0.8 & 0.5 & 439 & 13 & 1.0 & 0.5 & 440 & 16 & 1.2 & 0.4 & 491 & 21 & 2.3 & 0.9 \\
\hline $\mathrm{C}-\mathrm{IR}$ & $-32^{* *}$ & & 0.6 & & 1 & & 0.8 & & $-24^{* *}$ & & -0.6 & & $-27^{* *}$ & & -0.2 & \\
\hline
\end{tabular}

Note-IR, ignored repetition; C, control; AR, attended repetition. ${ }^{* *} p<.01$. 
Table 5

Mean Reaction Times (RTs, in Milliseconds), Standard Errors, and Percentages of Error (PEs) in the Prime Display As a Function of Attention Load in Experiments 3A and 3B

\begin{tabular}{cccccccccc}
\hline & \multicolumn{8}{c}{ Attention Load } \\
\cline { 2 - 7 } \cline { 7 - 10 } Experiment & RT & $S E$ & PE & $S E$ & & RT & $S E$ & PE & $S E$ \\
\hline 3A & 672 & 22 & 9.8 & 1.1 & & 812 & 26 & 31.0 & 1.1 \\
3B & 659 & 17 & 7.8 & 1.0 & & 802 & 19 & 28.8 & 2.2 \\
\hline
\end{tabular}

In Experiment 3A, the target images that were presented in the probe display were replaced with the words that described the target objects of the natural images at the basic level (e.g., the word bird for the image that included a bird, the word car for the image that included a car, and the word apple for the image that included an apple). Although the images were not presented repeatedly in Experiments 1 and 2, in Experiment 3A, the number of words that referred to objects in natural scenes was different in the three target categories. In fact, we used 69 words in this experiment. Also, the number of presentations for each word differed among the three target categories. All words were written in Japanese kana and were printed in white on a black background in Gothic font. The words were composed of approximately 3.2 letters. Each letter subtended a visual angle of about $1.4^{\circ}$ vertically and about $1.4^{\circ}$ horizontally.

Design and Procedure. The variables that were manipulated were attention load (low and high) and the relationship between prime display and probe display (IR, C, and AR). All were withinsubjects factors.

The procedure was identical to that of the natural scene categorization task in Experiment 2, except that the written-word stimuli were presented in the center of the probe display, rather than the natural images, and participants categorized them as animals, vehicles, or food. Also, the distractor image was not presented in the probe display.

\section{Results and Discussion}

The mean RTs and error rates as a function of the experimental conditions are presented in Table 5 for the prime display and Table 6 for the probe display. Trials with errors and trials preceded by an error on the prime display were excluded from the probe RT analysis, as were responses that deviated $2 S D$ s from an individual participant's means.

One-way (attention load: low vs. high) within-subjects ANOVAs were conducted on the mean RTs and error rates of the prime display. Both RTs and error rates showed the significant effects of attention load $[F(1,11)=15.43$, $M S_{\mathrm{e}}=7,608.28, p<.01$, and $F(1,11)=417.85, M S_{\mathrm{e}}=$ $6.45, p<.01$, respectively], indicating the adequate manipulation of attention load as expected. Also, a two-way within-subjects ANOVA on the mean RTs of the probe display indicated that the effect of relationship was significant $\left[F(1,11)=7.05, M S_{\mathrm{e}}=807.69, p<.05\right]$. The effect of attention load was marginally significant $[F(1,11)=$ 4.68, $\left.M S_{\mathrm{e}}=135.25, p=.052\right]$. The interaction between attention load and relationship was not significant $(F<1)$. A similar ANOVA on the error rates did not show any significant effect. Thus, on the basis of the RTs, we observed significant NP effects both in the low-attention-load condition and in the high-attention-load condition $(-19 \mathrm{msec}$ and $-24 \mathrm{msec}$, respectively). These results suggest that object categorization in natural scenes with minimal attention is made on the basis of not only low-level features (as found in Experiments 1 and 2), but also on the semantic information of the target categories.

Two-way within-subjects ANOVAs on RTs and error rates with variables of attention load (low vs. high) and relationship (AR vs. C) showed significant effects of relationship $\left[F(1,11)=21.50, M S_{\mathrm{e}}=2,276.03, p<.01\right.$, and $F(1,11)=12.41, M S_{\mathrm{e}}=12.98, p<.01$, respectively]. These results indicate the presence of a picture-primeword-target priming effect (see, e.g., Koivisto \& Revonsuo, 2000).

\section{EXPERIMENT 3B}

In Experiment 3A, because the number of words of each category was different, the results might have been contaminated. Therefore, we equated the number of probe word stimuli in Experiment 3B to confirm the results of Experiment 3A.

\section{Method}

Except for the aspects described below, the method was identical to that of Experiment 3A.

Participants. Twelve native Japanese undergraduate students of Nagoya University (6 men and 6 women, age range 18-19 years) participated in Experiment 3B. None had participated in the other experiments. All were right-handed and reported normal or corrected-to-normal visual acuity and normal color vision.

Stimuli and Procedure. We selected nine Japanese words for each target category, for a total of 27 words (e.g., rat for animal category, battleplane for vehicle category, and soup for food category). The frequencies of these words were counterbalanced. The words were composed of approximately 4.0 letters. Across the experiment, each word was presented equiprobably across three priming conditions.

The procedure was identical to that of Experiment $3 \mathrm{~A}$ except that the experiment consisted of 243 trials for each attention-load condition, because each word was presented equiprobably across

Table 6

Mean Reaction Times (RTs, in Milliseconds), Standard Errors, and Percentages of Error (PEs) in the Probe Display As a Function of Each Experimental Condition in Experiments $3 A$ and $3 B$

\begin{tabular}{|c|c|c|c|c|c|c|c|c|c|c|c|c|c|c|c|c|}
\hline \multirow[b]{3}{*}{ Condition } & \multicolumn{8}{|c|}{ Experiment $3 \mathrm{~A}$} & \multicolumn{8}{|c|}{ Experiment 3B } \\
\hline & \multicolumn{4}{|c|}{ Low } & \multicolumn{4}{|c|}{ High } & \multicolumn{4}{|c|}{ Low } & \multicolumn{4}{|c|}{ High } \\
\hline & RT & $S E$ & $\mathrm{PE}$ & $\overline{S E}$ & RT & $S E$ & $\mathrm{PE}$ & $\overline{S E}$ & RT & $S E$ & $\mathrm{PE}$ & $\overline{S E}$ & RT & $S E$ & $\mathrm{PE}$ & $S E$ \\
\hline IR & 554 & 18 & 6.3 & 1.4 & 549 & 17 & 5.4 & 1.1 & 575 & 17 & 6.2 & 1.5 & 591 & 21 & 5.1 & 1.2 \\
\hline $\mathrm{C}$ & 535 & 14 & 5.1 & 1.5 & 525 & 17 & 4.2 & 0.9 & 554 & 12 & 5.4 & 0.9 & 569 & 15 & 8.6 & 1.8 \\
\hline AR & 470 & 15 & 1.5 & 0.8 & 462 & 18 & 0.5 & 0.3 & 527 & 16 & 6.3 & 1.6 & 528 & 15 & 3.4 & 0.9 \\
\hline $\mathrm{C}-\mathrm{IR}$ & $-19^{*}$ & & -1.2 & & $-24^{*}$ & & -1.2 & & $-21^{*}$ & & -0.8 & & $-22^{*}$ & & 3.5 & \\
\hline
\end{tabular}

Note-IR, ignored repetition; $\mathrm{C}$, control; AR, attended repetition. ${ }^{*} p<.05$. 
three priming conditions. Thus, there were 18 blocks, and each of the 27 words was presented 18 times across the experiment. Another difference was that in the IR and AR conditions, the word stimuli in the probe display did not match the ignored objects and the attended objects in the natural images of the prime display, respectively, although they shared the same category at the superordinate level (e.g., the word rat for the image that included a lion, the word battleplane for the image that included a car, and the word soup for the image that included an apple). Also, in the $\mathrm{C}$ condition, word stimuli belonged to the remaining category that was not presented in the central and peripheral stimuli of the prime display.

\section{Results and Discussion}

The mean RTs and error rates as a function of the experimental conditions are presented in Table 5 for the prime display and Table 6 for the probe display. Trials with errors and trials preceded by an error on the prime display were excluded from the probe RT analysis, as were responses that deviated $2 S D$ s from an individual participant's means.

One-way (attention load: low vs. high) within-subjects ANOVAs were conducted on the mean RTs and error rates of the prime display. Both RTs and error rates showed significant effects of attention load $\left[F(1,11)=44.66, M S_{\mathrm{e}}=\right.$ $2,731.20, p<.01$, and $F(1,11)=123.59, M S_{\mathrm{e}}=21.41$, $p<.01$, respectively], indicating the adequate manipulation of attention load as expected. Also, a two-way withinsubjects ANOVA on the mean RTs of the probe display indicated that the effect of relationship was significant $\left[F(1,11)=6.14, M S_{\mathrm{e}}=885.87, p<.05\right]$. However, the effect of attention load and the interaction between attention load and relationship were not significant $[F(1,11)=$ 2.03, $M S_{\mathrm{e}}=1,456.78, p=.18$, and $F<1$, respectively]. Thus, we observed significant NP effects both in the lowattention-load condition and in the high-attention-load condition ( $-21 \mathrm{msec}$ and $-22 \mathrm{msec}$, respectively). A similar ANOVA conducted on the error rate data did not reveal any significant effect. These results replicated the findings of Experiment 3A, suggesting that object categorization in natural scenes with minimal attention is made on the basis of not only the basic-representational level (such as car), but also on the superordinate category level (such as vehicle). In addition, these results indicate that the NP we observed was not sensitive to the difference in number of words among target categories and the difference in the number of presentations of each word.

Two-way within-subjects ANOVAs were performed on the RTs and error rates, with the variables being attention load (low vs. high) and relationship (AR vs. C). Results revealed significant effects of relationship $[F(1,11)=$ $13.21, M S_{\mathrm{e}}=1,038.63, p<.01$, and $F(1,11)=5.03$, $M S_{\mathrm{e}}=10.79, p<.05$, respectively]. These results demonstrate the within-categories picture-word priming effect (see, e.g., Hines, Sawyer, Dura, Gilchrist, \& Czerwinski, 1984; Koivisto \& Revonsuo, 2000).

\section{GENERAL DISCUSSION}

The main findings of the present study were the following: (1) target objects in natural scenes are categorized with minimal attention; (2) letters are not discriminated unless adequate attentional resources are available; (3) object categorization with minimal attention is specific to natural scenes and does not occur with line drawings; and (4) object categorization in natural scenes with minimal attention is made on the basis of the semantic information of the natural images at the representation levels, such as car and vehicle.

In our experiments, NP effects were always found in the natural scene categorization task irrespective of attentionload conditions. On the other hand, the NP effect occurred with letters and line drawings only in the low-attention-load condition. These results are consistent with the proposal of Li et al. (2002). More importantly, object categorization in natural scenes with minimal attention is found with an NP paradigm (Lavie \& Fox, 2000) in which participants did not need to respond to peripheral stimuli themselves and without massive training, which were confounds in Li et al.'s study. Thus, we propose that even if attentional resources cannot be utilized, objects in natural scenes are categorized as though adequate attentional resources are available, whereas letters and line drawings are not categorized unless adequate attention is available. In addition, we showed in Experiments 3A and 3B that object categorization in natural scenes with minimal attention is made on the basis of the semantic information of images. These results are consistent with those of Evans and Treisman (2005), who found that the mere detection and access to the minimal semantic information of objects in natural images did not require attentional resources, whereas the identification and detailed scrutiny of target objects in natural images required attentional resources. The NP effects we observed could be based on the unbound features of natural images that contain the minimal semantic information needed to categorize them (cf. Evans \& Treisman, 2005) at the basic and superordinate levels (see, e.g., Jolicœur, Gluck, \& Kosslyn, 1984; Murphy \& Wisniewski, 1989; Rosch, Mervis, Gray, Johnson, \& Boyes-Braem, 1976), because it is unlikely that the objects in the to-be-ignored natural images were perceived in detail.

One could argue that the participants consciously looked at the peripheral stimuli, despite instructions to ignore them. However, this possibility should be dismissed for the following reason. If the participants had looked at the peripheral stimuli in the prime display, the RTs in the IR condition would be expected to be shorter than those in the $\mathrm{C}$ condition, because the target on the probe display had the same identity as the distractor in the previous prime display. Thus, one would expect to observe a positive repetition priming effect. However, in our experiments, the data showed significant NP effects or the absence of positive repetition priming and picture-prime--word-target priming. Therefore, we can conclude that the participants did not consciously look at the peripheral stimuli. Lachter, Forster, and Ruthruff (2004) showed that 110-msec prime duration produced a significant priming effect even if the prime was presented in the irrelevant location where the target did not occur. Lachter et al.'s findings suggested that for the 110-msec prime duration, participants could shift their attention to the irrelevant location. Although the 
240-msec prime duration in our study means that participants could shift their attention to the peripheral stimuli in the low-attention-load condition, the robust effect of attention load would make it less plausible that participants could shift their attention to the peripheral stimuli in the high-attention-load condition as well as the low-attentionload condition.

Also, some might insist that although the RT data of the letters and natural scenes in the AR condition seemed comparable, those of the IR and $\mathrm{C}$ conditions were considerably longer by roughly $150 \mathrm{msec}$ for the scenes, raising the possibility of additional processes. Neill and his colleagues (e.g., Neill, 1997; Neill \& Mathis, 1998; Neill $\&$ Valdes, 1992) indicated that the NP effect is due to the possibility that a longer probe-processing time affords a greater likelihood of retrieving past (i.e., prime display) distractor information. We would argue that the difference in RT is due to the difference in the visual search task for letter discrimination versus natural scene categorization. It is known that people can process simple stimuli (e.g., letters) more quickly than complex stimuli (e.g., natural scenes). We observed robust repetition priming effects both in the letter discrimination and in the natural scene categorization, and the magnitude of priming effects appeared similar. However, it is possible that the RT data in the AR condition showed a floor effect when participants performed the letter discrimination task, and such a floor effect might explain the difference between the RTs of the letter discrimination and those of the natural scene categorization tasks. On the other hand, if the presence of the NP effect is due to the longer RTs in the natural scene categorization in comparison with the letter discrimination task, then we would expect the presence of the NP effect in the high-attention-load condition for categorizing line drawings, because the RTs in the line-drawing categorization were longer than those in the letter discrimination task. However, the line-drawing categorization task did not demonstrate an NP effect in the high-attention-load condition. Considering the results of Experiments 1 and 2, we reject the hypothesis put forth by Neill and colleagues and conclude that the NP with natural scenes that occurred in the high-attention-load condition was due to the specificity of the natural images and not to the difference of length of the RTs in the natural scene categorization versus the letter discrimination.

Two theories have been postulated to explain NP: an inhibition theory (see, e.g., Houghton \& Tipper, 1994; Neill, 1977; Tipper, 1985; Tipper \& Cranston, 1985; Tipper \& Driver, 1988; Yee, 1991) and an episodic trace theory (e.g., Neill \& Valdes, 1992; Neill, Valdes, Terry, \& Gorfein, 1992). According to the inhibition explanation, when individuals have to attend selectively to a stimulus or stimulus dimension, their attention mechanisms emphasize the target element and simultaneously suppress the representations of task-irrelevant information. This inhibition view addresses the benefit of highlighting the target on the prime trial and the increased difficulty of using the suppressed representations shortly thereafter on the probe trial.
The episodic trace theory emphasizes memory retrieval as opposed to an inhibitory mechanism. Interference is considered to result from the response conflict between the "do not respond" tag attached to the ignored prime item and the "respond" tag attached to the target probe item (the same item). When the target probe is encountered, retrieval of recent relevant information occurs, but the item most likely to be retrieved (the ignored prime item) carries a tag that disrupts responding to the same item, which is now the target probe.

Both the inhibitory theory and the episodic trace theory can adequately explain the NP effect present for natural scenes in our high-attention-load condition. According to the theory of inhibition, the ignored natural images were processed with minimal or less attention and inhibited. Because a target on the probe display had the same identity as the distractor in the previous prime display, the NP could also be explained using the episodic trace theory. For the same reason, the NP effects observed in the lowattention-load condition of the letter discrimination, line drawing, and natural scene categorization tasks could be explained by either the inhibition or the episodic trace theory. However, the NP effects in Experiments 3A and 3B are better explained by the inhibition theory, because the target on the probe display did not have the same identity as the distractor in the previous prime display.

Although it is well known that NP is increased by the number of repetitions of a stimulus across trials (see, e.g., Grison \& Strayer, 2001; Malley \& Strayer, 1995; Neill \& Joordens, 2002; Strayer \& Grison, 1999), we argue that this repetition effect is not important in the NP procedure used in our study, because the advantage of natural scenes over letters and line drawings is so robust that the NP effects of natural scenes are observed with minimal attentional resources. On the other hand, DeSchepper and Treisman (1996) found robust NP with fairly complex nonsense shapes presented uniquely, so that some wonder whether the advantage of natural scenes with minimal attention might be due to the novelty of the stimuli. Indeed, the natural scenes were more novel than the letters and line drawings in our study. If the novelty of the scenes accounted for the advantage of the natural scenes, then we should have observed NP for line drawings in comparison with letters, because line drawings appear less frequently than letters and were more novel for the participants than were the letters. In Experiment 2, however, there was no NP effect for the line drawings. Therefore, the NP effect observed for the natural scenes in our study is not attributable to the novelty of the stimuli.

Why didn't we observe an NP effect in the letter discrimination and line-drawing categorization tasks under the condition of less attention? One possibility is that our visual system may be more attuned to well-learned stimuli when fewer attentional resources are available. Fei-Fei, VanRullen, Koch, and Perona (2005) pointed out that well-learned stimuli (e.g., an upright letter and a natural scene) can be processed when attentional resources are few, whereas this is not the case for less-learned stimuli (e.g., a randomly rotated letter and fixed rotated letter). 
Their proposal may explain some of our findings, considering that peripheral line drawings were not recognized in the high-attention-load condition. However, it is inconsistent with our finding that the NP priming effect was not observed in the (upright) letter discrimination task with less attention. This inconsistency might reflect the greater task difficulty of discriminating a target letter among the searched letters in our study, in comparison with Fei-Fei et al.'s (2005) task.

Given our results and the previous studies of natural scene categorization (e.g., Fei-Fei et al., 2005; Li et al., 2002), it seems true that target objects in natural scenes are categorized with minimal attention. However, it remains unclear what kind of model best explains the process of natural scene recognition with minimal attention, although the following may account for the processing of complex stimuli such as natural scenes.

Bar $(2003,2004)$ proposed a hypothetical model for the rapid visual processing of natural scene categorization. According to his model, the visual system rapidly detects low-frequency information ("coarse information") in natural scenes. This blurred information is believed to be projected to the prefrontal cortex (PFC), where possible candidates for the target objects are selected. This blurred information is also projected to the parahippocampal cortex (PHC), where the visual system selects the most likely context for the natural images. A few candidates for the target objects and the context frame are projected to the inferior temporal cortex (ITC), where the target object in the natural scene is identified. Such a line of processes has been hypothesized as underlying the recognition of natural scenes. Recent studies appear to be consistent with this hypothesis (Davenport \& Potter, 2004; Gordon, 2004). Our results may be explained by Bar's hypothesis. Participants might extract "coarse information" from the peripheral natural scene on the prime display even if they did not have attentional resources allocated to the peripheral stimulus. This coarse information would be projected to the PFC and PHC, where a few candidates for the target objects and the context frame would be selected, which then would be projected to the ITC. This process would generate the categorization of natural scene on the prime display with minimal attention. Hence, this information would affect the participants' subsequent responses to the probe display, including the occurrence of the NP effect.

A second explanation is possible, given the findings of VanRullen et al. (2004), who reported that targets do not pop out with natural scenes, even though they can be discriminated from distractors when attention is occupied elsewhere. Conversely, they showed that certain targets do pop out among distractors, and that they need undivided attention to be effectively discriminated from distractors when presented in isolation (rotated L vs. +, depth-rotated cubes). They proposed that attentional resources exist along the two independent dimensions of visual search and dual-task performance. According to their explanation, natural scene categorization is a serial task in visual search, but is a preattentive task in dual-task performance.
Our results are also consistent with VanRullen et al.'s (2004) framework. Some might wonder that if participants can categorize natural scenes with minimal attentional resources, why did our results demonstrate the presence of an attention-load effect on searching among natural scenes in the prime display? The hypothesis proposed by VanRullen et al. is helpful in explaining this attention-load effect. Specifically, the participants could respond to one or five central natural scenes with a serial process in visual search and simultaneously categorize a peripheral natural scene with minimal attention as a preattentive process in dual task. VanRullen et al. argued that the two modes of visual processing might be due to the existence of selective neuronal populations and the difference in size of the neuronal receptive fields (Desimone, Moran, \& Spitzer, 1988). In our experiments, it is possible that individuals cannot consciously process low-level features and semantic information of natural images simultaneously in visual search; meanwhile, in some types of dual task, they can unconsciously share this information regarding the natural images. It is conceivable that although the semantic information that a particular image contained an animal interfered with the information that other images contained a tree, sea, mountain, or snow in conscious processing, the semantic information that an ignored image contained a vehicle was shared, unconsciously, with the information that an image contained an animal. A recent study using fMRI showed that there were two areas that were involved in the decision of natural scenes with attention free and that were activated in conscious perception demanding attentional resources (Marois, Yi, \& Chun, 2004). Although a discrepancy between the attentive processing of natural scenes in visual search and the preattentive processing in a dual task has been found, this point remains under discussion. We intend to address this issue in our next study.

A third explanation of our results is the reverse hierarchy theory (RHT) that was proposed by Hochstein and his associates (Hershler \& Hochstein, 2005; Hochstein $\&$ Ahissar, 2002). According to this theory, visual information is processed by low-level perceptual mechanisms that feed forward through a hierarchy of low-level processes leading to increasingly complex but general representations. This feed-forward processing is believed to be implicit and does not require attention, whereas the topdown processing from higher to lower levels is explicit and needs attention. Unlike the previous two explanations, we think that the RHT does not adequately account for our results, because the higher order information of natural images (although it was limited to the representation of objects in images at the basic and superordinate level) was processed with minimal attention. So, this theory should be amended to address the effect of semantic information, which does not require attentional resources.

In conclusion, our study provides critical verification of the intriguing finding that target objects in natural scenes are categorized with minimal attention using an NP paradigm, on the basis of the semantic information of the images. In addition, our findings revealed that this result is specific to natural images and is not true of line draw- 
ings. We believe that our procedure is a more appropriate way to examine whether natural scenes are processed with fewer attentional resources, in comparison with the methodology of previous studies (e.g., Fei-Fei et al., 2005; Li et al., 2002), although our procedure does not tap the aspect of the rapidity of natural scene categorization. The findings of Evans and Treisman (2005) were not inconsistent with the feature integration theory (e.g., Treisman \& Gelade, 1980; Wolfe et al., 1989) in that the unbound features of the natural images, instead of the entire representation of objects, were extracted without attention. However, further experimentation should be carried out to determine whether the features that contain the minimal semantic information would be processed as well as lowlevel features, such as lines and colors. Although there is a computational approach (e.g., Torralba \& Oliva, 2003), our experimental findings are important in elucidating how human beings process the semantic information of natural scenes with minimal attention, and contribute to the development of a constructive and integrative model of natural scene recognition.

\section{AUTHOR NOTE}

We thank James E. Hoffman, Robert D. Gordon, Andrew Hollingworth, and Daniel J. Simons for their insightful comments on an earlier draft of this article. We also thank Shigeo Terashima for good advice about making stimuli. Correspondence concerning this article should be addressed to S. Otsuka or J. Kawaguchi, Department of Psychology, Graduate School of Environmental Studies, Nagoya University, Frocho, Chikusaku, Nagoya 464-8601, Japan (e-mail: s050307d@mbox .nagoya-u.ac.jp or kawaguchij@cc.nagoya-u.ac.jp).

\section{REFERENCES}

Bacon-Macé, N., Macé, M. J. M., Fabre-Thorpe, M., \& Thorpe, S. J. (2005). The time course of visual processing: Backward masking and natural scene categorisation. Vision Research, 45, 1459-1469.

BAR, M. (2003). A cortical mechanism for triggering top-down facilitation in visual object recognition. Journal of Cognitive Neuroscience, 15, 600-609.

BAR, M. (2004). Visual objects in context. Nature Reviews Neuroscience, 5, 617-629.

BRAUN, J. (1993). Shape-from-shading is independent of visual attention and may be a "texton." Spatial Vision, 7, 311-322.

BRAUN, J. (1994). Visual search among items of different salience: Removal of visual attention mimics a lesion in extrastriate area V4. Journal of Neuroscience, 14, 554-567.

Braun, J., \& Julesz, B. (1998). Withdrawing attention at little or no cost: Detection and discrimination tasks. Perception \& Psychophysics, 60, 1-23.

Braun, J., \& Sagi, D. (1990). Vision outside the focus of attention. Perception \& Psychophysics, 48, 45-58.

DavenPort, J. L., \& Potter, M. C. (2004). Scene consistency in object and background perception. Psychological Science, 15, 559-564.

DeSchepper, B., \& Treisman, A. (1996). Visual memory for novel shapes: Implicit coding without attention. Journal of Experimental Psychology: Learning, Memory, \& Cognition, 22, 27-47.

Desimone, R., Moran, J., \& Spitzer, H. (1988). Neural mechanisms of attention in extrastriate cortex of monkeys. In M. A. Arbib \& S. Amari (Eds.), Dynamic interactions in neural networks: Models and data (pp. 169-182). New York: Springer.

Evans, K. K., \& Treisman, A. (2005). Perception of objects in natural scenes: Is it really attention free? Journal of Experimental Psychology: Human Perception \& Performance, 31, 1476-1492.

Fei-Fei, L., VanRullen, R., Koch, C., \& Perona, P. (2005). Why does natural scene categorization require little attention? Exploring atten- tional requirements for natural and synthetic stimuli. Visual Cognition, 12, 893-924.

Fize, D., Fabre-Thorpe, M., Richard, G., Doyon, B., \& Thorpe, S. J. (2005). Rapid categorization of foveal and extrafoveal natural images: Associated ERPs and effects of lateralization. Brain \& Cognition, 59, $145-158$.

Gordon, R. D. (2004). Attentional allocation during the perception of scenes. Journal of Experimental Psychology: Human Perception \& Performance, 30, 760-777.

Grison, S., \& Strayer, D. L. (2001). Negative priming and perceptual fluency: More than what meets the eye. Perception \& Psychophysics, 63, 1063-1071.

Hershler, O., \& Hochstein, S. (2005). At first sight: A high-level pop out effect for faces. Vision Research, 45, 1707-1724.

Hines, D., Sawyer, P. K., Dura, J., Gilchrist, J., \& Czerwinski, M. (1984). Hemispheric asymmetry in use of semantic category information. Neuropsychologia, 22, 427-433.

Hochstein, S., \& Ahissar, M. (2002). View from the top: Hierarchies and reverse hierarchies in the visual system. Neuron, 36, 791-804.

Houghton, G., \& TipPer, S. P. (1994). A model of inhibitory mechanisms in selective attention. In D. Dagenbach \& T. H. Carr (Eds.), Inhibitory processes in attention, memory, and language (pp. 53-112). San Diego: Academic Press.

Johnston, W. A., \& DARK, V. J. (1986). Selective attention. Annual Review of Psychology, 37, 43-75.

Jolicceur, P., Gluck, M. A., \& Kosslyn, S. M. (1984). Pictures and names: Making the connection. Cognitive Psychology, 16, 243-275.

Kane, M. J., May, C. P., Hasher, L., RahHal, T., \& Stoltzfus, E. R. (1997). Dual mechanisms of negative priming. Journal of Experimental Psychology: Human Perception \& Performance, 23, 632-650.

Korvisto, M., \& Revonsuo, A. (2000). Semantic priming by pictures and words in the cerebral hemispheres. Cognitive Brain Research, 10, 91-98.

Lachter, J., Forster, K. I., \& RUthruff, E. (2004). Forty-five years after Broadbent (1958): Still no identification without attention. Psychological Review, 111, 880-913.

LAVIE, N. (1997). Visual feature integration and focused attention: Response competition from multiple distractor features. Perception \& Psychophysics, 59, 543-556.

Lavie, N., \& Fox, E. (2000). The role of perceptual load in negative priming. Journal of Experimental Psychology: Human Perception \& Performance, 26, 1038-1052.

Lee, D. K., Koch, C., \& Braun, J. (1999). Attentional capacity is undifferentiated: Concurrent discrimination of form, color, and motion. Perception \& Psychophysics, 61, 1241-1255.

Li, F. F., VanRullen, R., Koch, C., \& Perona, P. (2002). Rapid natural scene categorization in the near absence of attention. Proceedings of the National Academy of Sciences, 99, 9596-9601.

Malley, G. B., \& Strayer, D. L. (1995). Effect of stimulus repetition on positive and negative identity priming. Perception \& Psychophysics, 57, 657-667.

Marois, R., Yi, D. J., \& ChUn, M. M. (2004). The neural fate of consciously perceived and missed events in the attentional blink. Neuron, 41, 465-472.

MurPhy, G. L., \& WisniewsKi, E. J. (1989). Categorizing objects in isolation and in scenes: What a superordinate is good for. Journal of Experimental Psychology: Learning, Memory, \& Cognition, 15, 572-586.

NeILL, W. T. (1977). Inhibitory and facilitatory processes in selective attention. Journal of Experimental Psychology: Human Perception \& Performance, 3, 444-450.

NeILL, W. T. (1997). Episodic retrieval in negative priming and repetition priming. Journal of Experimental Psychology: Learning, Memory, \& Cognition, 23, 1291-1305.

Neill, W. T., \& Joordens, S. (2002). Negative priming and multiple repetition: A reply to Grison and Strayer (2001). Perception \& Psychophysics, 64, 855-860.

NeILl, W. T., \& MathIS, K. M. (1998). Transfer-inappropriate processing: Negative priming and related phenomena. In D. L. Medin (Ed.), The psychology of learning and motivation: Advances in research and theory (Vol. 38, pp. 1-44). San Diego: Academic Press.

NeILl, W. T., \& VALDES, L. A. (1992). Persistence of negative priming: 
Steady state or decay? Journal of Experimental Psychology: Learning, Memory, \& Cognition, 18, 565-576.

Neill, W. T., Valdes, L. A., Terry, K. M., \& Gorfein, D. S. (1992). Persistence of negative priming: II. Evidence for episodic trace retrieval. Journal of Experimental Psychology: Learning, Memory, \& Cognition, 18, 993-1000.

Rosch, E., Mervis, C. B., Gray, W. D., Johnson, D. M., \& BoyesBraem, P. (1976). Basic objects in natural categories. Cognitive Psychology, 8, 382-439.

SNODGRASS, J. G., \& VANDERWART, M. (1980). A standardized set of 260 pictures: Norms for name agreement, image agreement, familiarity, and visual complexity. Journal of Experimental Psychology: Human Learning \& Memory, 6, 174-215.

Sperling, G., \& Dosher, B. A. (1986). Strategy and optimization in human information processing. In K. R. Boff, L. Kaufman, \& J. P. Thomas (Eds.), Handbook of perception and human performance (pp. 1-65). New York: Wiley.

SPERling, G., \& Melchner, M. J. (1978). The attention operating characteristic: Examples from visual search. Science, 202, 315-318.

Strayer, D. L., \& Grison, S. (1999). Negative identity priming is contingent on stimulus repetition. Journal of Experimental Psychology: Human Perception \& Performance, 25, 24-38.

Thorpe, S., Fize, D., \& Marlot, C. (1996). Speed of processing in the human visual system. Nature, $\mathbf{3 8 1}, 520-522$.

TIPPER, S. P. (1985). The negative priming effect: Inhibitory priming by ignored objects. Quarterly Journal of Experimental Psychology, 37A, 571-590.

Tipper, S. P., \& Cranston, M. (1985). Selective attention and priming: Inhibitory and facilitatory effects of ignored primes. Quarterly Journal of Experimental Psychology, 37A, 591-611.

TIPPER, S. P., \& DrIVER, J. (1988). Negative priming between pictures and words in a selective attention task: Evidence for semantic processing of ignored stimuli. Memory \& Cognition, 16, 64-70.

Torralba, A., \& Oliva, A. (2003). Statistics of natural image categories. Network: Computation in Neural Systems, 14, 391-412.

Treisman, A. M., \& Gelade, G. (1980). A feature-integration theory of attention. Cognitive Psychology, 12, 97-136.

VAnRullen, R., \& КосH, C. (2003). Visual selective behavior can be triggered by a feed-forward process. Journal of Cognitive Neuroscience, 15, 209-217.
VAnRullen, R., Reddy, L., \& Koch, C. (2004). Visual search and dual tasks reveal two distinct attentional resources. Journal of Cognitive Neuroscience, 16, 4-14.

VAnRullen, R., \& Thorpe, S. J. (2001a). Is it a bird? Is it a plane? Ultra-rapid visual categorisation of natural and artifactual objects. Perception, 30, 655-668.

VAnRullen, R., \& Thorpe, S. J. (2001b). The time course of visual processing: From early perception to decision-making. Journal of Cognitive Neuroscience, 13, 454-461.

Wolfe, J. M., CaVe, K. R., \& Franzel, S. L. (1989). Guided search: An alternative to the feature integration model for visual search. Journal of Experimental Psychology: Human Perception \& Performance, 15, 419-433.

YeE, P. L. (1991). Semantic inhibition of ignored words during a figure classification task. Quarterly Journal of Experimental Psychology, 43A, 127-153.

\section{NOTES}

1. We think that it is worth examining whether natural scene categorization is accomplished in the situation in which participants are not required to respond directly to peripheral stimuli, although the results of Li et al. (2002) indicated that the training effect was not found in peripheral tasks (i.e., letters and color patterns) other than natural scene categorization.

2. Generally, in the negative priming experiment, participants are required to respond to the target while ignoring the distractor in each trial. What is important is the relationship between the distractor of trial $n$ and the target of trial $n+1$ in two successive trials. The stimuli of trials $n$ and $n+1$ are called prime and probe, respectively. In the control condition, no relation exists between the target and distractor of the prime and probe trials. On the other hand, in the ignored repetition condition, the distractor ignored in the prime trial is presented as the target on the subsequent probe trial. In general, RTs to the probe target in the ignored repetition condition are slower than those in the control condition. This delay is referred to as negative priming.

(Manuscript received February 13, 2006; revision accepted for publication March 12, 2007.) 